\title{
Implementation of an Automated Respiratory Amplitude Gating Technique for PET/CT: Clinical Evaluation
}

\author{
Guoping Chang ${ }^{1}$, Tingting Chang ${ }^{1}$, Tinsu Pan ${ }^{2}$, John W. Clark Jr. ${ }^{1}$, and Osama R. Mawlawi ${ }^{2}$ \\ ${ }^{1}$ Department of Electrical and Computer Engineering, Rice University, Houston, Texas; and ${ }^{2}$ Department of Imaging Physics, M.D. \\ Anderson Cancer Center, University of Texas, Houston, Texas
}

Amplitude gating techniques have recently been shown to be better at suppressing respiratory motion artifacts than phase gating. However, most commercial PET/CT scanners are equipped with phase gating capabilities only. The objective of this article was to propose and evaluate using patient studies an automated respiratory amplitude gating technique that could be implemented on current whole-body PET/CT scanners. A primary design feature of the proposed technique is to automatically match the respiratory amplitude captured during the CT scan with a corresponding amplitude during the PET scan. Methods: The proposed amplitude gating technique consists of a CT scan, followed by a list-mode PET scan. The CT scan was acquired while the patient's respiratory motion was recorded by a monitoring device that determined the respiratory motion amplitude captured during the CT scan. A program was designed to inject triggers into the PET list stream whenever the patient's respiration crossed a preset amplitude range determined by the captured amplitude during CT. To implement this proposed amplitude gating technique in whole-body PET/CT, a PET-first protocol was necessary to minimize the respiratory baseline drift between the CT and PET scans. In this implementation, a regular PET scan was first acquired over the patient's whole body but excluding the bed position that covered the lesion of interest. The whole-body CT scan was then acquired, followed by a list-mode PET acquisition over the bed position that covered the area of interest (lesion). The proposed amplitude gating technique was tested using 13 patients with 21 lung or thoracic tumors. Results: In the patient studies, the gated images - when compared with the ungated images - showed statistically significant improvements, with an average $27 \%$ and $28 \%$ increase in maximum and mean standardized uptake value, respectively, for all lesions. Furthermore, the tumors in the gated images showed better contrast using visual inspection and line profiles. Conclusion: The implementation of the proposed respiratory amplitude gating technique on current PET/ CT scanners is feasible, and amplitude-matched CT and PET data can be automatically generated using our proposed procedures without requiring patients to hold their breath or increase their radiation exposure.

Received Jul. 23, 2009; revision accepted Sep. 8, 2009.

For correspondence or reprints contact: Osama R. Mawlawi, Department of Imaging Physics, M.D. Anderson Cancer Center, University of Texas, 1515 Holcombe Blvd., Unit 1352, Houston, TX 77030.

E-mail: OMawlawi@di.mdacc.tmc.edu

COPYRIGHT () 2010 by the Society of Nuclear Medicine, Inc.
Key Words: amplitude gating; PET/CT; automation

J Nucl Med 2010; 51:16-24

DOI: 10.2967/jnumed.109.068759

$\mathbf{P}$ ET/CT is increasingly being used to facilitate the diagnosis, staging, and restaging of a wide variety of cancers (1-3). This is largely due to the ability of this imaging modality to provide anatomic and functional information about the underlying disease state. Furthermore, the addition of the CT component to PET scanners has also provided an efficient attenuation map, which has greatly reduced the total scan duration and increased the throughput of the scanner (4). However, the addition of CT to PET has also introduced some disadvantages. In a PET/ CT study, the CT scan captures the patient breathing cycle in a single state $(5,6)$, whereas the PET scan is usually acquired over many breathing cycles because of its longer acquisition time (7). This discrepancy introduces a mismatch between the CT and the PET images that results in mislocalization of small lesions and inaccurate quantification of the standardized uptake value (SUV) $(8-10)$. These effects eventually compromise the diagnostic accuracy of $\mathrm{PET} / \mathrm{CT}$ and might result in patient mismanagement.

To overcome respiratory motion artifacts in PET/CT, PET respiratory gating techniques have been proposed (11-15). These techniques can be divided into 2 categories: phase gating and amplitude gating (16), with phase gating being the only option available on current commercial PET/CT scanners. In phase gating, the respiratory cycle is divided into multiple phase ranges (or bins), and the acquired data are sorted into each phase range on the basis of their acquisition time within the respiratory cycle. This approach works well for patients with regular breathing but results in large errors in patients who have irregular respiration (frequency or amplitude) primarily because of the introduction of large amounts of motion in each bin (16). Recently, amplitude gating has been proposed as an alternative approach to phase gating (16). Rather than dividing the respiratory cycle into different phase ranges, amplitude gating divides the respiratory amplitude into different amplitude ranges. In this 
regard, motion artifacts in phase gating are suppressed with amplitude gating. However, amplitude gating is currently unavailable on any commercial PET/CT scanner.

Approaches to implement amplitude gating on commercial PET/CT scanners are currently being investigated by many research groups (17-20). Some of the suggested methods rely on 4-dimensional (4D) PET, whereby the acquired PET data are retrospectively sorted into multiple amplitude ranges using commercial respiratory gating devices. The corresponding reconstructed images are then also retrospectively registered to one another to generate a motion-free image that is characterized by a good signalto-noise ratio (SNR) $(17,18)$. Other methods, known as motion-incorporated reconstruction techniques, are focused on incorporating the motion information between the different bins of the 4D PET images into the statistical reconstruction algorithm to reduce motion artifacts while maintaining good SNR $(19,20)$. Both of these methods, however, require an additional 4D CT scan, which is characterized by increased patient radiation exposure, to attenuate-correct the corresponding 4D PET data. Furthermore, the implementation of these methods also relies on deformable image registration techniques that might not result in accurate image registration because of the low SNR of the different PET bins or inconsistency in respiratory motion between CT and PET images. Recently, deep-inspiration breath-hold (DIBH) techniques have been proposed as a variant of amplitude gating, without the need to register the 4D PET images or increase patient exposure (21-23). In DIBH, patients are requested to hold their breath at deep inspiration for a relatively short period while both the CT and PET data are acquired. PET data acquisition in this respiratory state is then repeated multiple times to accumulate a sufficient amount of counts per pixel $(21,22)$. The resultant multiple-PET datasets are then summed together to generate a motion-free sinogram, which is then attenuation-corrected by the corresponding CT image and eventually reconstructed into a motion-free image. The net result is a PET image that is matched in respiratory amplitude with a corresponding DIBH CT image. DIBH has been demonstrated to be feasible on current PET/CT scanners (21-23). However, a main disadvantage of this technique is ensuring patients' compliance with breath-holding for the specified time and amplitude in each respiratory session, particularly when the patients are at an increased state of anxiety because of their medical condition. Another disadvantage of DIBH is its extensive reliance on technologist-patient interaction to coordinate data acquisition during the multiple times the procedure is repeated to accumulate the necessary data, particularly with patients who have hearing or language barriers. Furthermore, recent studies have shown that approximately $60 \%$ of the lung cancer patients cannot perform the DIBH technique successfully (24).

In this article, we propose a novel approach to implement respiratory amplitude gating in whole-body PET/CT scan- ners that does not require any patient coaching or compliance with specific breathing conditions and maintains the advantages of DIBH, namely no increase in $\mathrm{x}$-ray exposure or deformable image registration. The main emphasis of this new approach was to perform respiratory amplitude gating with minimal patient and user interaction and at the same time minimize data postprocessing tasks. The proposed approach is similar to DIBH, except that the respiratory amplitude range during PET is automatically selected to match the breathing amplitude captured during the CT scan. In this approach, patients are allowed to breathe freely during the $\mathrm{CT}$ acquisition. The respiratory motion amplitude that is captured during the CT scan is then automatically used during PET in such a way that only PET data falling within a corresponding amplitude range are used to generate the final PET image. In this regard, the drawbacks of the 4D PET/CT acquisition and DIBH technique, such as the difficulty of 4D PET registration, high patient x-ray exposure, and patients' noncompliance, will be eliminated. The objective of this article was to describe how such a respiratory amplitude gating scheme could be automated in whole-body PET/CT scanners and evaluate its feasibility in current PET/CT scanners using patient studies.

\section{MATERIALS AND METHODS}

\section{Automatic Respiratory Amplitude Gating Approach}

Two goals must be achieved when automating the amplitude gating technique on current PET/CT scanners. First, the process must be done with minimal user interaction, and second, the respiratory motion amplitudes captured during the CT and PET scans should automatically match with one another.

To achieve these goals, we propose the following amplitude gating approach: acquire a CT scan, followed by a list-mode PET scan over the specific area of interest (usually the patient's torso), monitor the patient's breathing waveform during the CT scan and determine the amplitude of the breathing cycle when the CT scan reaches the area of interest (lesion location), and acquire a PET scan in list mode and extract only the PET data that are acquired when the patient's respiratory amplitude falls within a range determined by the breathing amplitude captured during the CT scan. In this regard, the motion amplitudes captured during the CT and PET scans are automatically matched with one another, without any technologist-patient interaction. Such a design paradigm, however, might result in a long PET session necessary to record a sufficient number of events, depending on the amplitude at which the CT captured the patient's breathing cycle (e.g., mid inspiration is characterized by a short transient time). Therefore, to reduce the overall scan duration, the PET scan is designed to be terminated when either 3 min worth of list-mode data are accumulated in the selected amplitude range or a maximum 10-min scan is reached. The use of 3 min worth of accumulated data was based on our standard scan duration per bed position at our institution.

To apply the proposed amplitude gating approach to wholebody PET/CT with multiple bed positions requires a modification of the standard protocol of a PET/CT scan. Rather than acquiring the usual whole-body CT scan, followed by a whole-body PET scan, the implementation of the proposed approach necessitates that the PET and CT scans corresponding to the area of interest 
(lesion) be temporally acquired as close as possible to one another. This is primarily to ensure that the patient's breathing amplitude pattern during CT remains similar to that during PET. On the basis of our experience as well as that of others, there exists a baseline drift in the respiratory waveform of most patients imaged with PET/CT. This drift, however, stabilizes within about $10 \mathrm{~min}$ from the time the patient is positioned on the PET/CT couch. Figure 1 shows an example of a breathing waveform without (Fig. 1A) and with (Fig. 1B) baseline drift. The breathing waveform in Figure 1B stabilized within a short time $(5-10 \mathrm{~min})$. In this regard, to minimize the effect of this drift on matching the respiratory amplitude during the CT and PET scans over the area of interest (lesion location), the proposed amplitude gating approach was based on a PET-first protocol as shown in Figure 2. In this design, the data acquisition is divided into the following 3 steps: a regular PET scan over the patient's whole body but excluding the bed position that covered the lung or thoracic lesion of interest, a regular whole-body CT scan, and a single-bed list-mode PET scan over the area of interest (lesion location). The bed position over the area of interest is skipped in the first step, to reduce the overall scan duration because the same bed position is acquired in the third step. The a priori knowledge of which bed position should be skipped can be derived from previous PET/CT scans of the patient or images from other diagnostic imaging modalities.
The baseline drift problem of the patient's breathing cycle is automatically resolved using this protocol, because the duration of the regular PET scan (step 1) usually takes 10-20 min (4-6 bed positions), which is long enough for the baseline drift to subside. Furthermore, the PET and CT scans over the lesion location in this design are acquired at close temporal proximity to one another to further minimize any remaining breathing variations.

The aim of this 3-step procedure was to capture the breathing amplitude during the CT scan and use it to select a corresponding amplitude range from the list-mode PET data, thereby ensuring matched breathing amplitude between the CT and the PET data. To capture the breathing amplitude during the $\mathrm{CT}$ scan requires recording the patient's respiratory waveform and determining the exact tumor location. In our approach, the respiratory waveform was recorded using a respiratory gating device (Anzai AZ-733V Respiratory Gating System; Anzai Medical Co.) (23), and the exact tumor location was determined from the reconstructed CT image. Both of these processes are synchronized a priori. In this regard, the breathing amplitude at the time the CT scan reached the area of interest (lesion location) could then be derived by correlating the tumor location with the recorded respiratory waveform. The respiratory amplitude captured during CT could then be matched to that of PET by coupling the Anzai device to a data-acquisition system (National Instruments) that had been programmed using
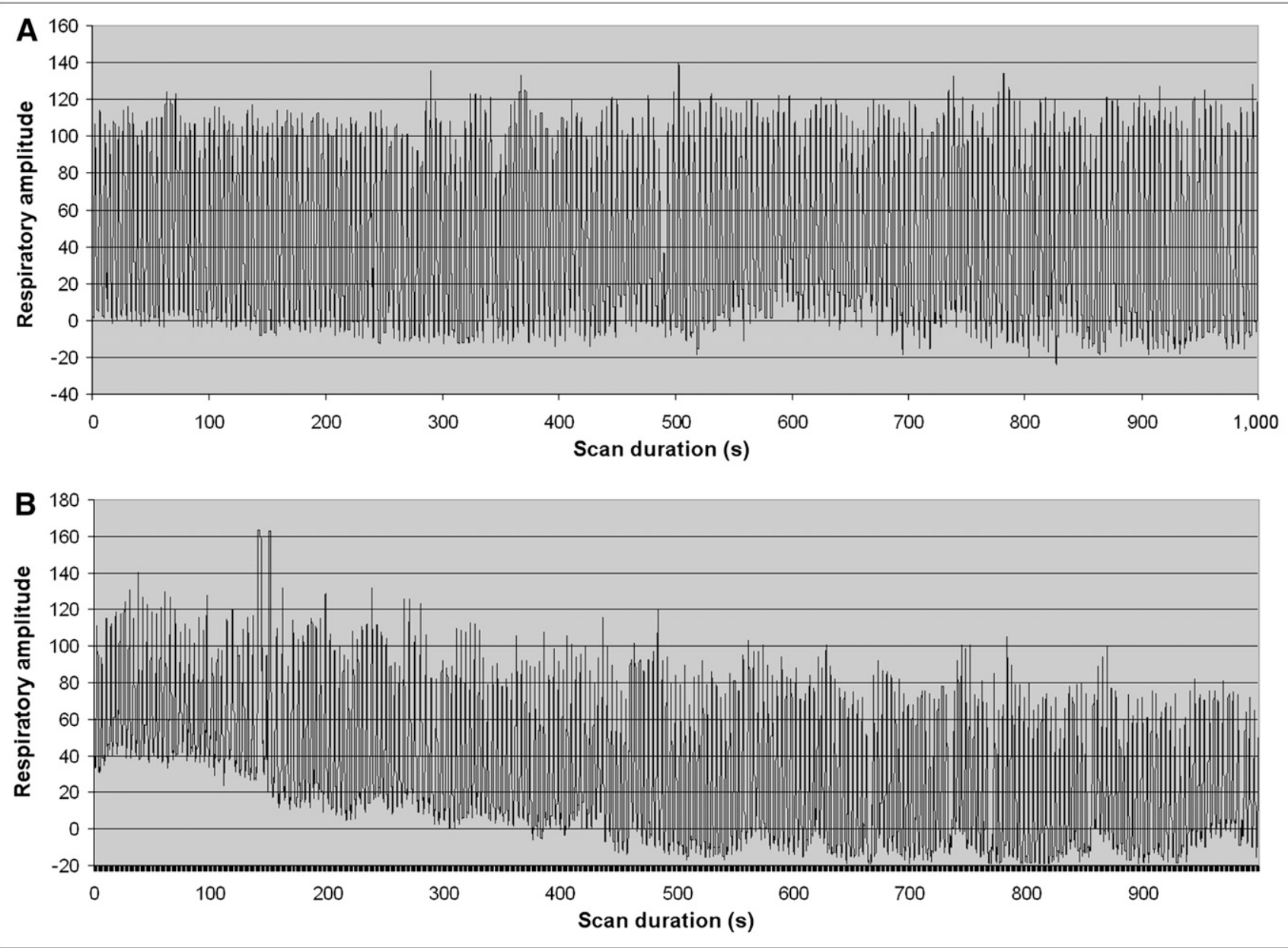

FIGURE 1. Breathing waveform without $(A)$ and with $(B)$ baseline drift. 


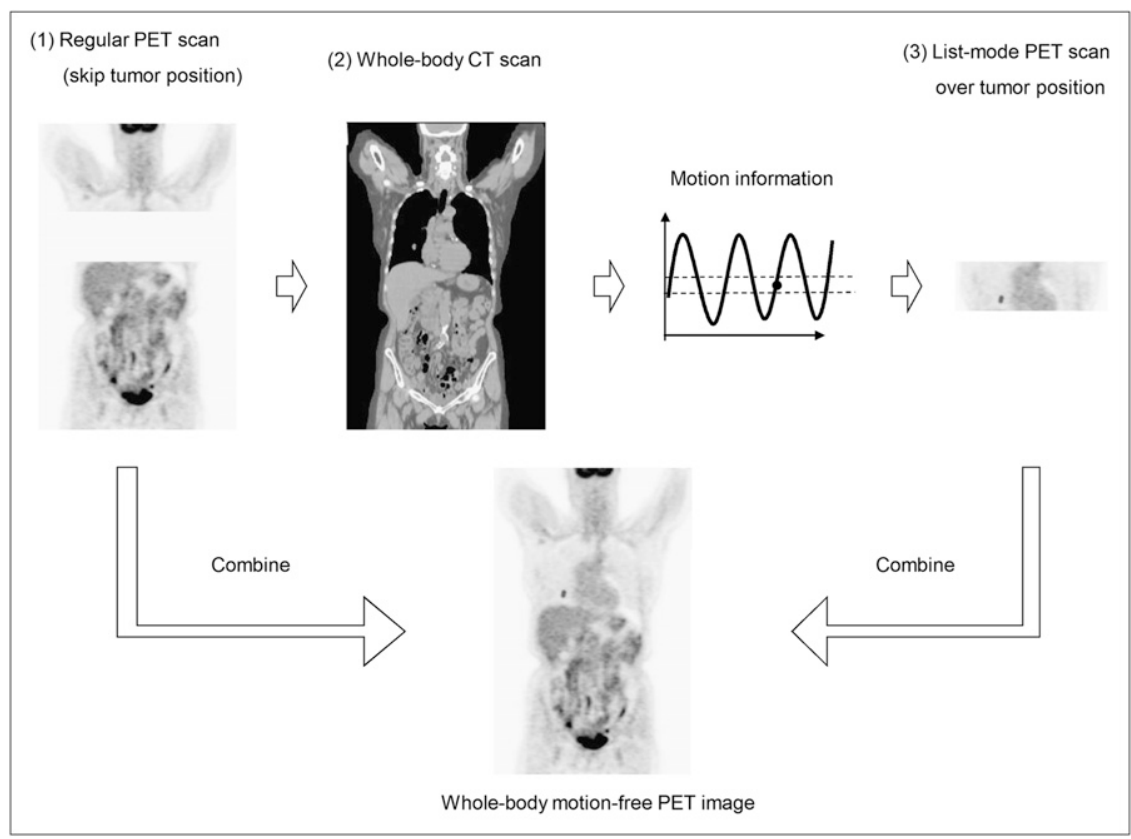

FIGURE 2. Procedures of proposed amplitude gating implementation.
LabVIEW (National Instruments) to inject triggers into the PET list stream whenever the patient's respiratory level crossed a $\pm 10 \%$ range of the captured amplitude during the CT scan (Fig. 3). This process continued until the end of the data acquisition.

After data acquisition, the PET list stream will contain alternating segments of data separated by triggers that were acquired within and outside the preset amplitude range (or gate) as shown in Figure 4. This list-mode data can then be either rebinned as a static scan or filtered in such a way as to generate a contiguous stream of PET list data acquired only within the preset amplitude range. This filtering process was necessary because the rebinning of the list-mode data could not be configured to select only events that were acquired within the preset amplitude range. In this regard, a list-resorting program was written in $\mathrm{C}$ and was used to resort the list data in such a way that all events acquired at different breathing cycles but within the preset amplitude range were placed contiguously and in a chronologic order in front of events that were acquired outside the selected amplitude range. The resorted list-mode file would then be processed by the data-rebinning function of the scanner to extract the portion of the data that was acquired only within the gated amplitude range. The amount of list-mode data to be extracted was determined from the output of the list-resorting program. Because the extracted PET data contained only information that was acquired when the respiratory waveform fell within the gate, the data could be directly reconstructed to generate an amplitude-gated PET image after attenuation correction.

The whole process of this amplitude gating implementation, except for the identification of the tumor location from the CT image and the configuration of the Anzai device to output triggers based on the selected amplitude range, was automatically performed using an in-house software program that did not require any user interference. Therefore, this amplitude gating approach can be implemented and automated on current commercial PET/ CT scanners. In this article, we tested the automation of this amplitude gating approach on 13 patients and evaluated the ability of the approach to reduce respiratory motion artifacts.

\section{PET/CT Scanner}

A Discovery RX PET/CT scanner (GE Healthcare) was used in this study. The PET gantry of this scanner consists of 24 rings of 630 detector crystals and has a transaxial field of view (FOV) of $70 \mathrm{~cm}$. The CT component of this scanner had a $50-\mathrm{cm}$ transaxial field of view. The description and performance characteristics of this PET/CT scanner have been published elsewhere (25). All data in the patient studies were acquired in 3-dimensional (3D) mode and were corrected for attenuation, random, scatter, and dead time and reconstructed using a 3D ordered-subset expectation maximization algorithm (2 iterations, 21 subsets).

\section{Patient Studies}

The objective of the patient studies was to test the proposed automation approach of the amplitude gating technique in clinical PET/CT patient studies. Institutional review board approval (MDACC IRB 2008-0851) was first acquired before the patient studies.

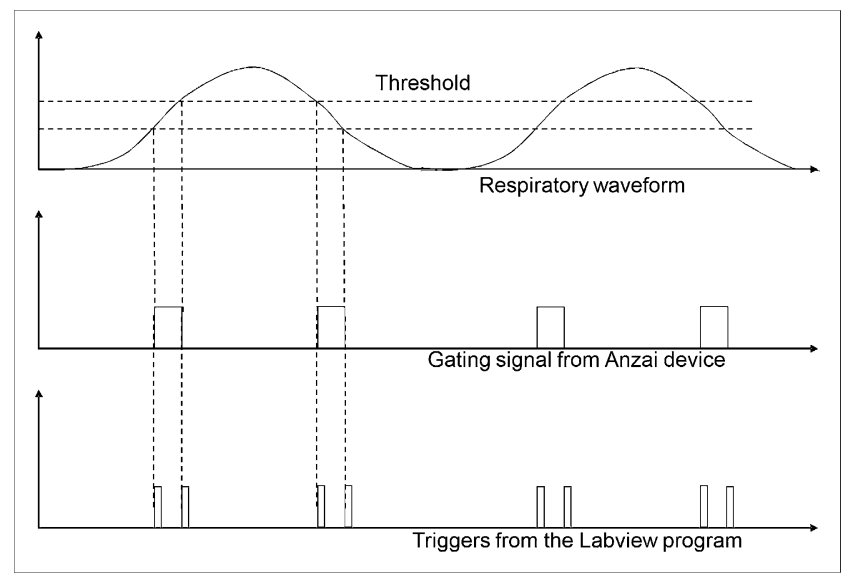

FIGURE 3. Relationship between respiratory waveform and triggers generated from LabVIEW program. 


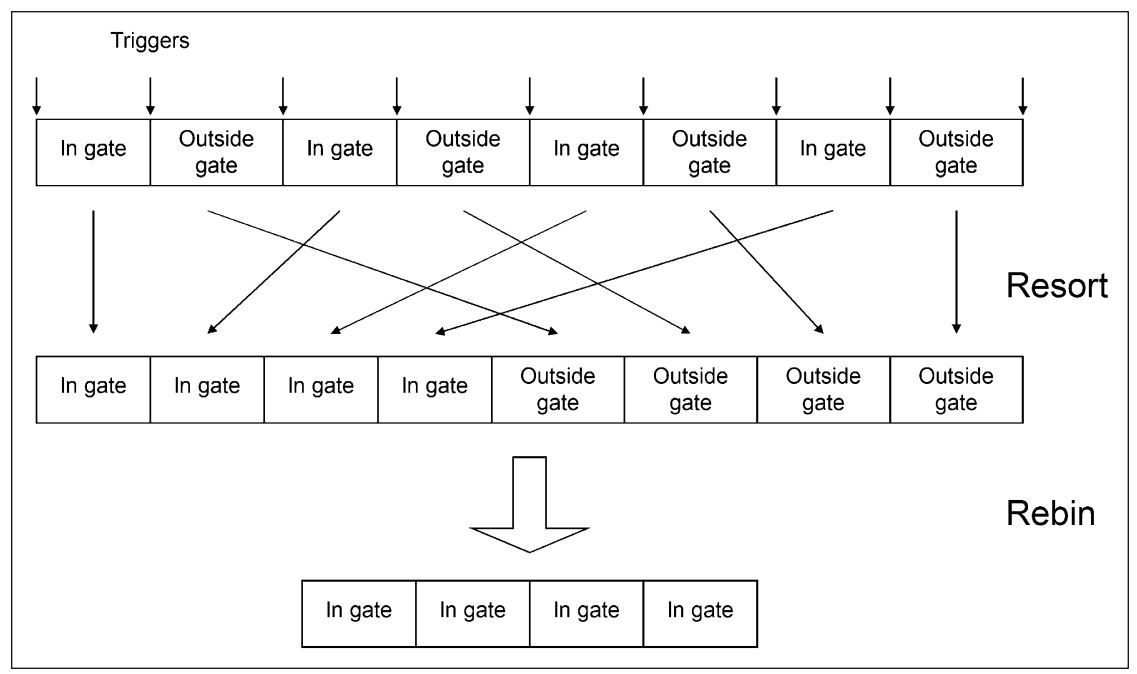

Thirteen patients ( 5 men and 8 women; mean age \pm SD, $64 \pm$ $9 \mathrm{y})$ referred for PET/CT evaluation of lung or thoracic lesions were selected to test the performance of the proposed amplitude gating approach. All patients fasted for $4 \mathrm{~h}$ before being injected intravenously with $296-444 \mathrm{MBq}(8-12 \mathrm{mCi})$ of ${ }^{18}$ F-FDG. Imaging started 60-90 min after injection. The same amplitude gating procedures as described above were applied in these studies, and the whole imaging process consisted of a regular PET scan covering $4-6$ bed positions (excluding the bed position that covered the tumor of interest), depending on the patient's height, a wholebody CT scan, and a list-mode PET scan of 1 bed position that covered the tumor location. During the list-mode PET scan over the tumor location, the Anzai device was configured to monitor the total time the tumor fell within the preset amplitude range and stopped the scan when either $3 \mathrm{~min}$ worth of data were accumulated in the selected amplitude range or a maximum of $10 \mathrm{~min}$ of scan duration was reached. This stopping condition resulted in an average of $8.3 \mathrm{~min}$ of list-mode PET scanning and a 2.6-min duration within the gate for all of the 13 patients. The information on the patients and their scan conditions are summarized in Table 1.
The acquired regular PET scan for each patient was then reconstructed using a 3D ordered-subset expectation maximization algorithm (2 iterations, 21 subsets) after attenuation correction by the CT image. In addition, the list-mode PET data were first postprocessed and then reconstructed using the same algorithm to generate the amplitude-gated image. This amplitudegated image was combined with the regular PET image to generate a motion-free whole-body PET image. For comparison purposes, the same list-mode data were rebinned as a 3-min static scan (without resorting), reconstructed using the same algorithm, and combined with the regular PET image to generate an ungated whole-body PET image. The amplitude-gated and ungated images were compared with one another using visual inspection and line profiles. The maximum SUV, mean SUV, lesion SNR, and lesion volume were determined for all lesions. The mean SUV and lesion volume of each lesion were calculated on the basis of a region of interest determined using a $40 \%$ maximum SUV threshold. The lesion SNR was defined as the mean SUV of the lesion divided by the SUV SD of a region of interest drawn in the lung. A statistical $t$ test was performed to evaluate the significance of the improve-

\section{TABLE 1. Summary of Patient Characteristics and PET/CT Conditions}

\begin{tabular}{|c|c|c|c|c|c|c|c|}
\hline Patient no. & Sex & Age $(y)$ & No. of tumors & Lesion site & $\begin{array}{l}\text { No. of FOV in } \\
\text { regular PET scan }\end{array}$ & $\begin{array}{l}\text { List-mode } \\
\text { PET scan } \\
\text { duration (min) }\end{array}$ & $\begin{array}{c}\text { Accumulated } \\
\text { time within } \\
\text { gate (min) }\end{array}$ \\
\hline 1 & $\mathrm{~F}$ & 65 & 2 & Left lower and right lower robe & 5 & 10 & 2.4 \\
\hline 2 & M & 81 & 2 & Left lower and right lower robe & 5 & 10 & 1.8 \\
\hline 3 & $\mathrm{~F}$ & 66 & 1 & Upper liver & 6 & 10 & 2.2 \\
\hline 4 & M & 64 & 1 & Upper liver & 5 & 8.9 & 3.0 \\
\hline 5 & $\mathrm{~F}$ & 47 & 2 & Right middle lobe, close to rib & 6 & 5.6 & 3.0 \\
\hline 6 & $\mathrm{~F}$ & 54 & 1 & Left lower lobe & 6 & 10 & 2.1 \\
\hline 7 & $\mathrm{~F}$ & 57 & 1 & Left upper lobe & 6 & 10 & 2.2 \\
\hline 8 & $\mathrm{~F}$ & 60 & 1 & Left middle lobe & 4 & 5.8 & 3.0 \\
\hline 9 & $\mathrm{~F}$ & 68 & 2 & Left upper and lower lobe & 5 & 8.7 & 3.0 \\
\hline 10 & M & 65 & 3 & Right upper lobe and upper liver & 5 & 4.3 & 3.0 \\
\hline 11 & M & 71 & 1 & Upper liver & 6 & 5.6 & 3.0 \\
\hline 12 & $\mathrm{~F}$ & 71 & 2 & Middle rib & 4 & 10 & 2.4 \\
\hline 13 & M & 69 & 2 & Upper liver & 6 & 8.8 & 3.0 \\
\hline Average & - & 64 & - & - & - & 8.3 & 2.6 \\
\hline
\end{tabular}


ment in maximum SUV, mean SUV, lesion SNR, and lesion size on the gated images versus the ungated images.

\section{RESULTS}

The maximum and mean SUV, lesion SNR, and volume for all 21 tumors are summarized in Table 2 for both amplitude-gated and ungated images. The percentage differences (\% diff) of the SUV, lesion SNR, and volume between the gated and the ungated images are also calculated. This table shows that the maximum and mean SUV and lesion SNR are improved in the amplitude-gated images versus the ungated images. The average improvement for the maximum SUV (range, 17\%-62\%), mean SUV (range, $13 \%-77 \%$ ), and lesion SNR (range, -3.4\%$81 \%$ ) was $26.8 \%, 28 \%$, and $26.3 \%$, respectively. The improvement in lesion SNR was primarily due to the improvement of the mean SUV of the tumor. The $t$ test showed that the improvement in SUV and lesion SNR were statistically significant $(P<0.05)$ in the gated versus the ungated images. The lesion volumes, as shown in Table 2 , also decreased by $37.1 \%$ on average as a result of reducing the motion blurring using the proposed amplitude gating technique. The $t$ test also showed that the decrease in tumor volume in the gated image, compared with the ungated images, was statistically significant $(P<0.05)$.

The results from 2 lung cancer patients are shown in Figure 5A. The tumors on the ungated and the amplitudegated PET/CT fused images are indicated (arrows). The first patient had a non-small cell lung cancer (NSCLC) lesion in the lower left lobe and a large mass in the right lobe. The comparison between the ungated and the gated images clearly shows that the mismatch problem between the CT and the PET due to the respiratory motion had been resolved in the amplitude-gated image. This result confirms that the gated PET image was acquired during the same respiratory amplitude captured in the CT scan. The maximum and mean SUV of the NSCLC lesion improved by $29.5 \%$ and $27.0 \%$, respectively, after using the proposed amplitude gating technique. The second patient had a collapsed lung with 2 NSCLC tumors close to each other in the right middle lobe. The images in Figure 5A (bottom) clearly show that the 2 tumors in the ungated PET images do not match their corresponding positions on the CT image (arrows), whereas in the gated images, the PET and CT information match well with one another. The maximum SUV for the 2 NSCLC lesions improved by $16.6 \%$ and $21.4 \%$, respectively. Line profiles across the 3 NSCLC tumors for the 2 patients are shown in Figure 5B, respectively. The CT anatomic structures of the 3 tumors are superimposed on these line profiles for comparison. These line profiles support our conclusion that the PET and CT images match well with one another when the proposed amplitude gating approach is applied.

\section{DISCUSSION}

In this article, we described a procedure to automate an amplitude gating approach that could be applied in wholebody PET/CT. This approach enabled the automatic matching of the respiratory amplitude captured during the CT and PET scans, without requiring the patients to hold their breath or maintain any specific breathing pattern. Furthermore, this amplitude gating technique retained the same

\begin{tabular}{|c|c|c|c|c|c|c|c|c|c|c|c|c|c|}
\hline \multirow{2}{*}{$\begin{array}{c}\text { Lesion } \\
\text { no. }\end{array}$} & \multirow{2}{*}{$\begin{array}{c}\text { Patient } \\
\text { no. }\end{array}$} & \multicolumn{3}{|c|}{$\mathrm{SUV}_{\max }$} & \multicolumn{3}{|c|}{$S U V_{\text {mean }}$} & \multicolumn{3}{|c|}{ SNR } & \multicolumn{3}{|c|}{ Lesion volume $\left(\mathrm{cm}^{3}\right)$} \\
\hline & & Ungated & Gated & $\%$ diff & Ungated & Gated & $\%$ diff & Ungated & Gated & $\%$ diff & Ungated & Gated & $\%$ diff \\
\hline 1 & 1 & 6.78 & 8.52 & 25.7 & 4.22 & 5.30 & 25.6 & 102.9 & 171.0 & 66.1 & 2.15 & 1.47 & 31.6 \\
\hline 2 & 1 & 3.39 & 3.95 & 16.5 & 1.71 & 2.34 & 36.8 & 41.7 & 75.5 & 81.0 & 5.67 & 1.37 & 75.8 \\
\hline 3 & 2 & 11.86 & 13.96 & 17.7 & 7.84 & 9.26 & 18.1 & 137.0 & 132.3 & -3.4 & 1.37 & 1.27 & 7.3 \\
\hline 4 & 2 & 6.32 & 10.26 & 62.3 & 3.85 & 6.85 & 77.9 & 67.3 & 97.9 & 45.5 & 1.47 & 0.59 & 59.9 \\
\hline 5 & 3 & 8.71 & 10.58 & 21.5 & 5.34 & 6.53 & 22.3 & 84.9 & 87.2 & 2.7 & 11.54 & 8.02 & 30.5 \\
\hline 6 & 4 & 13.44 & 16.06 & 19.5 & 8.43 & 9.91 & 17.6 & 89.5 & 109.9 & 22.8 & 11.25 & 9.19 & 18.3 \\
\hline 7 & 5 & 4.53 & 5.28 & 16.6 & 2.67 & 3.19 & 19.5 & 33.2 & 33.8 & 1.6 & 1.66 & 1.07 & 35.5 \\
\hline 8 & 5 & 5.37 & 6.52 & 21.4 & 3.39 & 4.45 & 31.3 & 42.2 & 47.1 & 11.7 & 2.94 & 1.85 & 37.1 \\
\hline 9 & 6 & 3.12 & 4.04 & 29.5 & 2.00 & 2.54 & 27.0 & 35.9 & 44.5 & 23.7 & 10.86 & 4.59 & 57.7 \\
\hline 10 & 7 & 12.32 & 15.28 & 24.0 & 6.89 & 8.24 & 19.6 & 91.5 & 125.7 & 37.4 & 10.17 & 6.65 & 34.6 \\
\hline 11 & 8 & 3.78 & 4.73 & 25.0 & 2.02 & 2.52 & 24.7 & 28.1 & 34.1 & 21.4 & 20.1 & 12.3 & 38.8 \\
\hline 12 & 9 & 3.01 & 4.09 & 36.0 & 1.62 & 2.06 & 26.9 & 17.6 & 18.9 & 7.5 & 22.0 & 7.9 & 64.1 \\
\hline 13 & 9 & 2.59 & 3.38 & 30.6 & 1.39 & 1.72 & 24.1 & 15.1 & 15.8 & 4.6 & 59.2 & 26.8 & 54.7 \\
\hline 14 & 10 & 17.89 & 25.41 & 42.1 & 11.26 & 15.99 & 41.9 & 178.2 & 243.4 & 36.6 & 1.47 & 1.37 & 6.8 \\
\hline 15 & 10 & 17.05 & 20.04 & 17.5 & 9.88 & 13.17 & 33.3 & 156.3 & 200.5 & 28.2 & 1.47 & 1.17 & 20.4 \\
\hline 16 & 10 & 3.48 & 4.43 & 27.3 & 1.89 & 2.23 & 18.2 & 29.9 & 33.9 & 13.5 & 21.70 & 13.5 & 37.8 \\
\hline 17 & 11 & 14.17 & 17.86 & 26.0 & 7.79 & 10.32 & 32.5 & 89.1 & 120.7 & 35.4 & 6.94 & 4.11 & 40.8 \\
\hline 18 & 12 & 7.07 & 8.56 & 21.1 & 3.91 & 4.80 & 22.7 & 92.4 & 128.9 & 39.5 & 1.56 & 1.17 & 25.0 \\
\hline 19 & 12 & 5.41 & 6.46 & 19.3 & 3.13 & 3.53 & 12.9 & 73.9 & 94.8 & 28.2 & 2.54 & 2.05 & 19.3 \\
\hline 20 & 13 & 11.74 & 14.60 & 24.4 & 7.38 & 8.58 & 16.2 & 93.8 & 105.7 & 12.7 & 6.94 & 5.18 & 25.4 \\
\hline 21 & 13 & 2.84 & 3.96 & 39.4 & 1.50 & 2.09 & 39.1 & 19.1 & 25.7 & 35.0 & 18.5 & 7.73 & 58.2 \\
\hline Average & & - & - & 26.8 & - & - & 28.0 & - & - & 26.3 & - & - & 37.1 \\
\hline
\end{tabular}



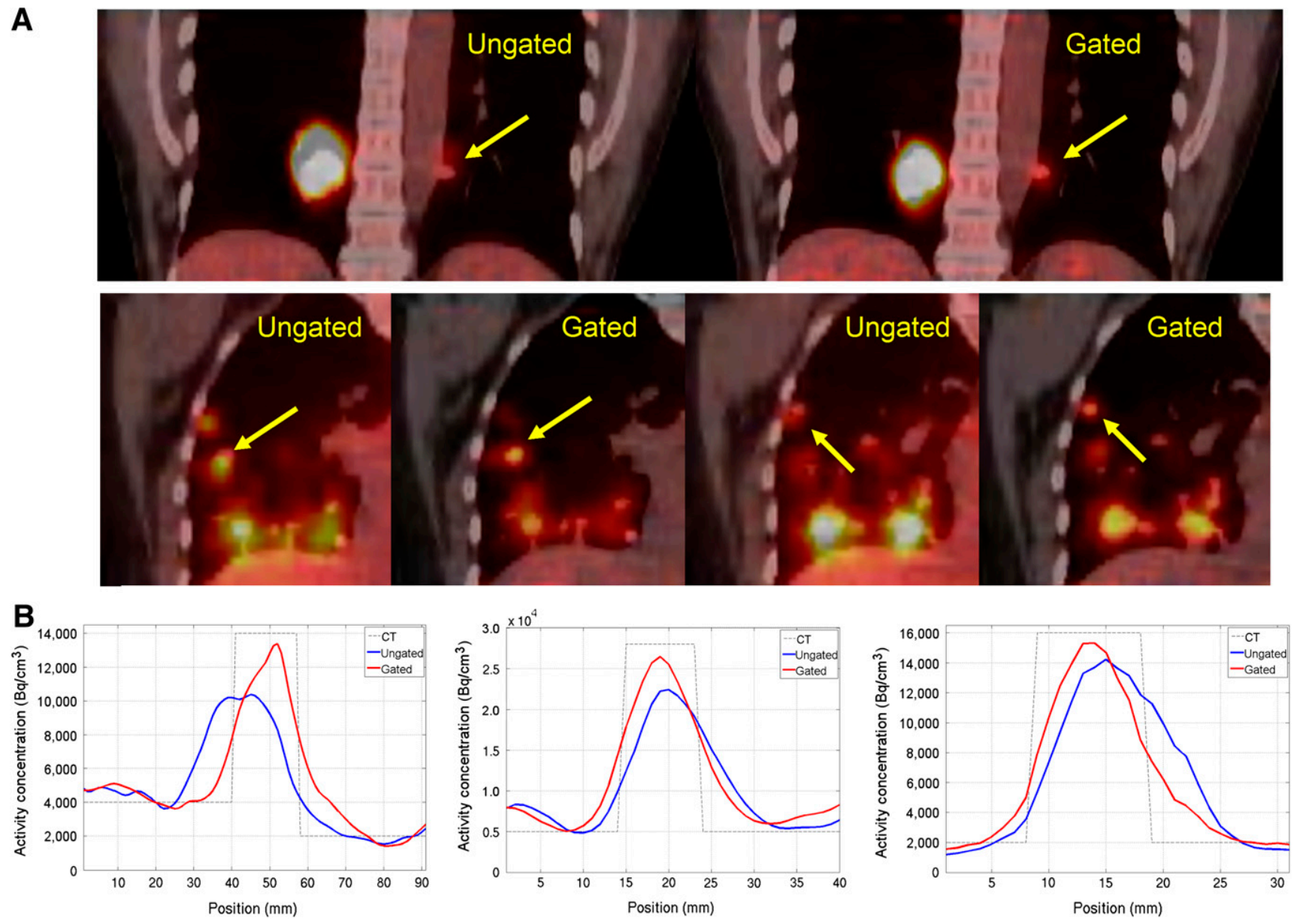

FIGURE 5. (A) Results from 2 lung cancer patients are shown. Tumors are indicated by arrows. (B) Line profiles across 3 tumors are shown.

advantages of DIBH with no additional x-ray exposure or deformable image registration. To test the performance of this approach, 13 patient studies were analyzed. The results from these studies showed that tumors on the amplitudegated images matched well with their locations on the CT images and exhibited higher maximum and mean SUV when compared with the ungated images. Therefore, this new implementation of respiratory amplitude gating is feasible in clinical conditions and has the capability of reducing respiratory motion artifacts in PET images.

One of the objectives of this article was to automate the proposed amplitude gating approach, with minimal user interactions. In our suggested approach, the acquisitions of the regular PET scan and the whole-body CT scan were already automated because they followed the same setup as a standard PET/CT protocol in current PET/CT scanners. The remaining processes that need to be automated are the determination of the lesion motion amplitude during the CT scan and the selection of a corresponding amplitude range during the list-mode PET acquisition. Both of these tasks have been automated using an in-house software program while requiring only that the technologists identify (using the mouse) the tumor location in the CT image. The output of this software program is then manually entered into the Anzai device to subsequently inject triggers into the PET list stream. The list-mode PET data acquisition is then manually initiated and is also manually terminated when either 3 min worth of PET list-mode data are accumulated in the selected amplitude range or a maximum of $10 \mathrm{~min}$ of scan duration is reached. The manual termination can be further automated if the manufacturer allows the injection of a trigger from the gating device (Anzai system) to stop the acquisition and terminate the scan. In this regard, the whole process of our amplitude gating implementation can be fully automated, except for the manual delineation of the physical tumor location, manual entry of the amplitude range in the Anzai gating system, and manual initiation and termination of the list-mode PET scan.

The data acquired from the list-mode PET scan need to be first filtered before they can be reconstructed to generate an amplitude-gated image. This filtering process is performed using a list-resorting program, followed by the data-rebinning process of the PET/CT scanner. One might argue that this process can be completed using a 1-step 
approach whereby the data acquired outside the amplitude range can be directly removed from the list-mode data and only events that fall within the specified amplitude range are left. This suggestion, however, affects the total counting rate, eventually resulting in different random, scatter, and dead-time correction, and affects SUV in the reconstructed PET image, which can eventually lead to inaccurate diagnosis.

In our proposed amplitude gating approach, a retrospective resorting of the acquired data is required before that can be reconstructed. To minimize this postprocessing task of the data, we suggest that the manufacturer of the scanner directly store data acquired within and outside the gate in 2 different memory locations in a manner similar to phase binning, for which data in different phases are stored in different bins. In this case, the gated PET data can then be directly reconstructed (prospectively) to generate an amplitude-gated PET image, thereby further shortening the implementation of amplitude gating on PET/CT scanners.

In our approach, the regular PET scan (step 1) is designed to skip 1 bed position over the area of interest (lesion location). This skipping step is realized by setting the scan duration of the bed position over the area of interest to the minimum allowable time $(1 \mathrm{~s})$, because current PET/CT scanners cannot be configured to skip over any bed position during a whole-body PET scan. Other approaches to realize the skipping step are possible, such as acquiring 2 different PET scans over the patient's upper and lower body, separated by a gap representing the skipped bed position. The most optimal method, however, would be to require the manufacturer to allow the skipping of any bed position as determined from the scout scan.

Another hurdle with the bed-skipping step is that it requires a priori knowledge about the rough location of the tumor of interest. This information can be derived from either previous PET/CT scans or other diagnostic images of the patient. For first-time patients, however, it is difficult to identify the location of the lesion of interest in advance. One method to solve this problem is to skip over the patient's whole torso during the regular PET scan (2-3 bed positions using $1 \mathrm{~s}$ per bed position) because this region is the area most affected by respiratory motion. A whole-body $\mathrm{CT}$ is then acquired, followed by a list-mode PET acquisition over the entire torso. This method, however, is characterized by relatively longer scan duration because it requires additional bed positions for the list-mode PET scan. Another method to determine the tumor location for first-time patients is not to skip any bed positions but rather acquire a whole-body regular PET scan using 3 min per bed position. The center of the tumor can then be determined from either the regular whole-body PET image or the CT image and is then used to determine the motion amplitude that will be used during the list-mode PET scan. This method, however, increases the total scan duration by $3 \mathrm{~min}$ because of the extra bed position during the regular PET scan that would have otherwise been skipped.
One disadvantage of our proposed approach is the relatively long scan duration of the list-mode PET scan and shorter time accumulated in the selected amplitude range. The long scan duration is because only a small portion of the acquired PET data falls within the preset amplitude range. To reduce the total scan duration, the list-mode PET scan was designed to be stopped when either $3 \mathrm{~min}$ worth of data were accumulated or a maximum of $10 \mathrm{~min}$ of scan duration was reached. In this case, the overall scan duration increased by only $7 \mathrm{~min}$. Our patient studies showed that the additional scan duration for our proposed amplitude gating approach was on average $5.3 \mathrm{~min}$ (range, 1.3-7.0 $\mathrm{min}$ ) and therefore did not greatly affect the standard PET/CT protocol. Furthermore, the limitation of a 10-min overall scan duration during the listmode PET acquisition could also result in a shorter time accumulated in gate, especially when a transient amplitude (e.g., mid inspiration) is captured during the CT scan. Our patient studies, however, have shown that the average accumulated time was $2.6 \mathrm{~min}$, with a range of $1.8-3 \mathrm{~min}$, suggesting that on average about 3 min within gate could be achieved if the total scan duration was $10 \mathrm{~min}$. Approaches to increase the time accumulated in-gate during the list-mode PET scan to make it exactly 3 min include further increasing the scan duration of the list-mode PET acquisition or acquiring data during the patient's end-expiration, which is characterized by higher duration ratio in the respiratory cycles. This second approach, however, will require the patients to hold their breath at end-expiration during the CT scan to capture the same motion amplitude as that during the PET scan. Such an approach, on the other hand, will require patient compliance and technologist-patient interaction, which will have the same problems as the DIBH technique and capture the lungs in a collapsed state that might affect the detectability of small lesions.

\section{CONCLUSION}

In this article, we proposed a novel approach to automate the respiratory amplitude gating implementation in wholebody PET/CT. In this approach, the motion amplitude captured during the CT scan was automatically matched with a corresponding amplitude during the PET data acquisition. The results from the patient studies show that this approach can be successfully implemented in current PET/CT scanners and has the ability to suppress respiratory motion artifacts.

\section{ACKNOWLEDGMENT}

This work was supported in part by GE Healthcare.

\section{REFERENCES}

1. Lardinois D, Weder W, Hany TF, et al. Staging of non-small-cell lung cancer with integrated positron-emission tomography and computed tomography. $N$ Engl J Med. 2003;348:2500-2507.

2. Schöder H, Larson SM, Yeung HW. PET/CT in oncology: integration into clinical management of lymphoma, melanoma, and gastrointestinal malignancies. J Nucl Med. 2004;45(suppl 1):72-81. 
3. von Schulthess GK. Positron emission tomography versus positron emission tomography/computed tomography: from "unclear" to "new-clear" medicine. Mol Imaging Biol. 2004;6:183-187.

4. Kinahan PE, Townsend DW, Beyer T, Sashin D. Attenuation correction for a combined 3D PET/CT scanner. Med Phys. 1998;25:2046-2053.

5. Beyer T, Antoch G, Muller S, et al. Acquisition protocol considerations for combined PET/CT imaging. J Nucl Med. 2004;45(suppl 1):25S-35S.

6. Blodgett TM, McCook BM, Federle MP. Positron emission tomography/computed tomography: protocol issues and options. Semin Nucl Med. 2006;36:157-168.

7. Mawlawi O, Kappadath SC, Pan T, Rohren E, Macapinlac HA. Factors affecting quantification in PET/CT imaging. Current Med Imaging Rev. 2008;4:34-45.

8. Sarikaya I, Yeung HW, Erdi Y, Larson SM. Respiratory artefact causing malpositioning of liver dome lesion in right lower lung. Clin Nucl Med. 2003;28:943-944.

9. Nehmeh SA, Erdi YE, Ling CC, et al. Effect of respiratory gating on quantifying PET images of lung cancer. $J$ Nucl Med. 2002;43:876-881.

10. Erdi YE, Nehmeh SA, Pan T, et al. The CT motion quantitation of lung lesions and its impact on PET-measured SUVs. J Nucl Med. 2004;45:1287-1292.

11. Nehmeh SA, Erdi YE, Ling CC, et al. Effects of respiratory gating on quantifying PET images of lung caner. J Nucl Med. 2002;43:876-881.

12. Boucher L, Rodrigue S, Lecomte R, Benard F. Respiratory gating for 3dimensional PET of the thorax: feasibility and initial results. J Nucl Med. 2004;45:214-219.

13. El Naqa I, Low DA, Bradley JD, Vicic M, Deasy JO. Deblurring of breathing motion artifacts in thoracic PET images by deconvolution methods. Med Phys. 2006;33:3587-3600.

14. Lamare F, Cresson T, Savean J, et al. Respiratory motion correction for PET oncology applications using affine transformation of list mode data. Phys Med Biol. 2007;52:121-140.
15. Bundschuh RA, Martinez-Moller A, Essler M, Nekolla SG, Ziegler SI, Schwaiger M. Local motion correction for lung tumors in PET/CT: first results. Eur J Nucl Med Mol Imaging. 2008;35:1981-1988.

16. Dawood M, Buther F, Lang N, Schober O, Schafers KP. Respiratory gating in positron emission tomography: a quantitative comparison of different gating schemes. Med Phys. 2007;34:3067-3075.

17. Klein GJ, Reutter BW, Ho MH, Reed JH, Huesman RH. Real-time system for respiratory-cardiac gating in positron tomography. IEEE Trans Nucl Sci. 1998; 45:2139-2143.

18. Schafers KP, Stegger L. Combined imaging of molecular function and morphology with PET/CT and SPECT/CT: image fusion and motion correction. Basic Res Cardiol. 2008;103:191-199.

19. Li T, Thorndyke B, Schreibmann E, Yang Y, Xing L. Model-based image reconstruction for four-dimensional PET. Med Phys. 2006;33:1288-1298.

20. Qiao F, Pan T, Clark JW, Mawlawi OR. A motion-incorporated reconstruction method for gated PET studies. Phys Med Biol. 2006;51:3769-3783.

21. Nehmeh SA, Erdi YE, Meirelles GSP, et al. Deep-inspiration breath-hold PET/ CT of the thorax. J Nucl Med. 2007;48:22-26.

22. Meirelles GSP, Erdi YE, Nehmeh SA, et al. Deep-inspiration breath-hold PET/ CT: clinical findings with a new technique for detection and characterization of thoracic lesions. J Nucl Med. 2007;48:712-719.

23. Kawano T, Ohtake E, Inoue T. Deep-inspiration breath-hold PET/CT of lung cancer: maximum standardized uptake value analysis of 108 patients. $\mathrm{J} \mathrm{Nucl}$ Med. 2008;49:1223-1231.

24. Keall PJ, Mageras GS, Balter JM, et al. The management of respiratory motion in radiation oncology report of AAPM Task Group 76. Med Phys. 2006;33:3874-3900.

25. Kemp BJ, Kim C, Williams JJ, Ganin A, Lowe VJ. NEMA NU 2-2001 performance measurements of an LYSO-based PET/CT system in 2D and 3D acquisition modes. J Nucl Med. 2006;47:1960-1967. 